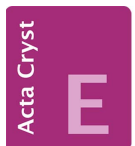

CRYSTALLOGRAPHIC COMMUNICATIONS

ISSN 2056-9890

Received 7 October 2015

Accepted 22 October 2015

Edited by M. Weil, Vienna University of Technology, Austria

Keywords: crystal structure; hydrazine; iron phosphate; isotypism

Supporting information: this article has supporting information at journals.iucr.org/e
CCDC reference: 1432700

\section{Crystal structure of hydrazine iron(III) phosphate, the first transition metal phosphate containing hydrazine}

\author{
Renald David
}

Laboratoire de Réactivité et Chimie des Solides (LRCS), Université de Picardie Jules Verne, CNRS UMR 7314, 33 rue Saint Leu, 80039 Amiens, France. *Correspondence e-mail: renald.david@u-picardie.fr

The title compound, poly[ $\left(\mu_{2}\right.$-hydrazine $)\left(\mu_{4}\right.$-phosphato)iron(III)], $\left[\mathrm{Fe}\left(\mathrm{PO}_{4}\right)\right.$ $\left.\left(\mathrm{N}_{2} \mathrm{H}_{4}\right)\right]_{n}$, was prepared under hydrothermal conditions. Its asymmetric unit contains one $\mathrm{Fe}^{\mathrm{III}}$ atom located on an inversion centre, one $\mathrm{P}$ atom located on a twofold rotation axis, and two $\mathrm{O}$, one $\mathrm{N}$ and two $\mathrm{H}$ atoms located on general positions. The $\mathrm{Fe}^{\mathrm{III}}$ atom is bound to four $\mathrm{O}$ atoms of symmetry-related $\mathrm{PO}_{4}$ tetrahedra and to two $\mathrm{N}$ atoms of two symmetry-related hydrazine ligands, resulting in a slightly distorted $\mathrm{FeO}_{4} \mathrm{~N}_{2}$ octahedron. The crystal structure consists of a three-dimensional hydrazine/iron phoshate framework whereby each $\mathrm{PO}_{4}$ tetrahedron bridges four $\mathrm{Fe}^{\mathrm{III}}$ atoms and each hydrazine ligand bridges two $\mathrm{Fe}^{\mathrm{III}}$ atoms. The $\mathrm{H}$ atoms of the hydrazine ligands are also involved in moderate $\mathrm{N}-\mathrm{H} \cdots \mathrm{O}$ hydrogen bonding with phosphate $\mathrm{O}$ atoms. The crystal structure is isotypic with the sulfates $\left[\mathrm{Co}\left(\mathrm{SO}_{4}\right)\left(\mathrm{N}_{2} \mathrm{H}_{4}\right)\right]$ and $\left[\mathrm{Mn}\left(\mathrm{SO}_{4}\right)\left(\mathrm{N}_{2} \mathrm{H}_{4}\right)\right]$.

\section{Chemical context}

During the last century, transition metal phosphates have been studied intensively not only for their rich crystal- and magneto-chemistry (Kabbour et al., 2012), but also for their various potential applications. For example, $\mathrm{NH}_{4} M^{\mathrm{II}} \mathrm{PO}_{4} \cdot \mathrm{H}_{2} \mathrm{O}$ phases, where $M$ is a transition metal, are used as pigments for protective paint finishes on metals, as fire retardants in paints
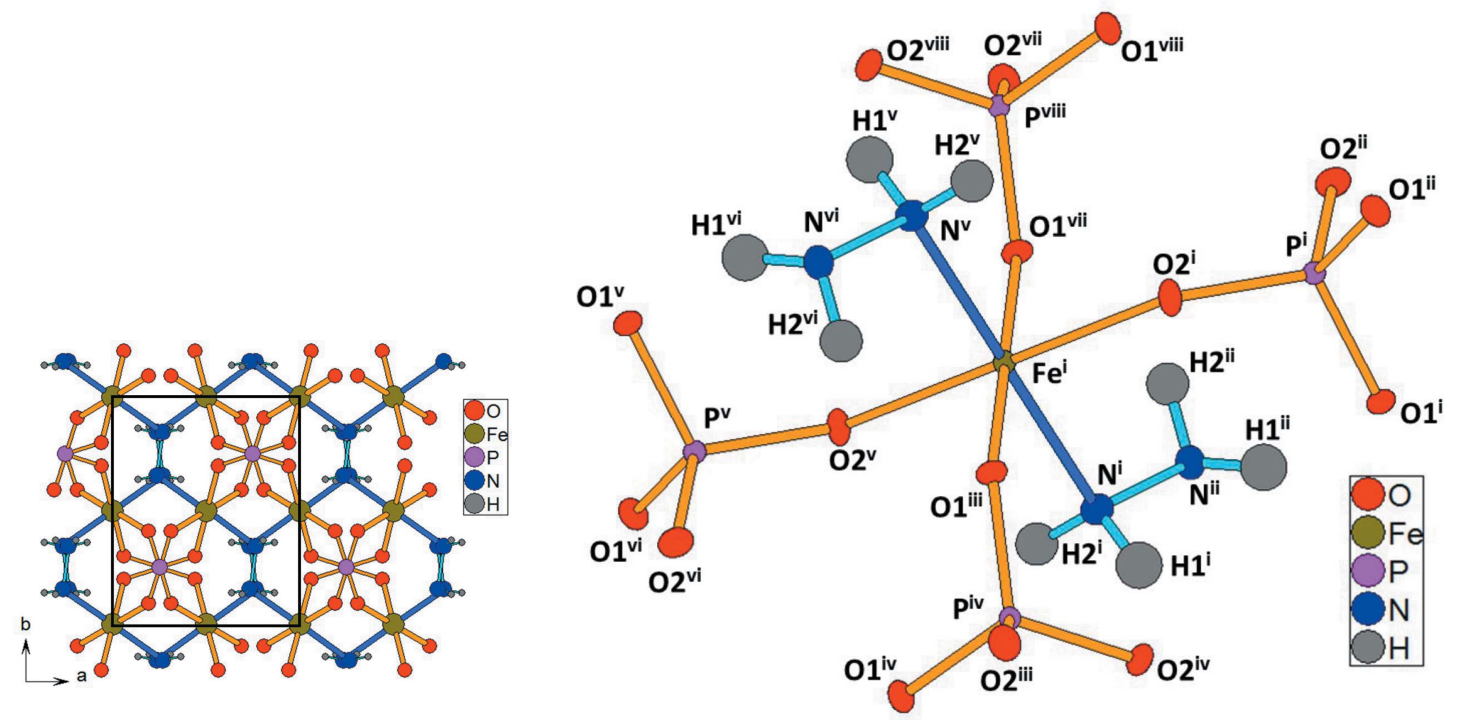

Figure 1

The coordination environment of the $\mathrm{Fe}^{\mathrm{III}}$ atom in the structure of $\left[\mathrm{Fe}\left(\mathrm{PO}_{4}\right)\left(\mathrm{N}_{2} \mathrm{H}_{4}\right)\right]$. Displacement ellipsoids are drawn at the $50 \%$ probability level. [Symmetry codes: (i) $x, y, z$; (ii) $0.5-x, 0.5-y$; (iii) $-x$, $y+0.5,0.5-z$; (iv) $x+0.5,-y, 0.5-z$; (v) $-x,-y,-z$; (vi) $x+0.5, y+0.5$, $-z$; (vii) $x, 0.5-y, z+0.5$; (viii) $0.5-x, y, z+0.5$.] 
Table 1

Hydrogen-bond geometry $\left(\AA,^{\circ}\right)$.

\begin{tabular}{lllll}
\hline$D-\mathrm{H} \cdots A$ & $D-\mathrm{H}$ & $\mathrm{H} \cdots A$ & $D \cdots A$ & $D-\mathrm{H} \cdots A$ \\
\hline $\mathrm{N}-\mathrm{H} 1 \cdots \mathrm{O} 1^{\mathrm{i}}$ & $0.85(3)$ & $2.36(2)$ & $3.086(2)$ & $144(2)$ \\
$\mathrm{N}-\mathrm{H} 1 \cdots \mathrm{O} 2^{\text {ii }}$ & $0.85(3)$ & $2.27(3)$ & $2.974(2)$ & $141(2)$ \\
$\mathrm{N}-\mathrm{H} 2 \cdots \mathrm{O} 1^{\text {iii }}$ & $0.85(3)$ & $2.19(3)$ & $2.873(2)$ & $137(2)$ \\
\hline
\end{tabular}

Symmetry codes: (i) $-x+\frac{1}{2},-y+\frac{1}{2}, z$; (ii) $x+\frac{1}{2},-y,-z+\frac{1}{2}$; (iii) $-x+\frac{1}{2}, y, z-\frac{1}{2}$.

and plastics but may also be applied as catalysts, fertilizers and magnetic devices (Erskine et al., 1944; Bridger et al., 1962; Barros et al., 2006; Ramajo et al., 2009). More recently, it was demonstrated by Goodenough and co-workers that in electrodes the presence of $\mathrm{PO}_{4}$ groups results in higher positive potentials (Padhi et al., 1997), leading to an intensive research on $\mathrm{LiFePO}_{4}$, one of the most promising materials for the new generation of Li batteries (Ouvrard et al., 2013).

\section{Structural commentary}

The structure of the title compound, $\left[\mathrm{Fe}\left(\mathrm{PO}_{4}\right)\left(\mathrm{N}_{2} \mathrm{H}_{4}\right)\right]$, is isotypic with the sulfates $\left[\mathrm{Co}\left(\mathrm{SO}_{4}\right)\left(\mathrm{N}_{2} \mathrm{H}_{4}\right)\right]$ and $\left[\mathrm{Mn}\left(\mathrm{SO}_{4}\right)\left(\mathrm{N}_{2} \mathrm{H}_{4}\right)\right]$ (Jia et al., 2011). The Fe ${ }^{\mathrm{III}}$ atom is bound to four $\mathrm{PO}_{4}$ tetrahedra and to two $\mathrm{N}$ atoms of hydrazine ligands, resulting in a slightly distorted $\mathrm{FeO}_{4} \mathrm{~N}_{2}$ octahedron (Fig. 1). The crystal structure consists of a three-dimensional network made up of $\mathrm{Fe}^{\mathrm{III}}$ atoms which are interconnected through neutral hydrazine $\left(\mathrm{N}_{2} \mathrm{H}_{4}\right)$ ligands and phosphate $\left(\mathrm{PO}_{4}{ }^{3-}\right)$ anions (Fig. 2). If the phosphate and sulfate structures are isotypic, the presence of phosphate implies an oxidation state of + III for the transition metal compared to + II for the sulfate analogues. The replacement of sulfate for phosphate leads to a change in the coordination sphere of the metal. These differences are mainly associated with the metal-oxygen bond lengths. The average $\mathrm{Fe}^{\mathrm{III}}-\mathrm{O}$ bond length is $1.97 \AA$ for $\left[\mathrm{Fe}\left(\mathrm{PO}_{4}\right)\left(\mathrm{N}_{2} \mathrm{H}_{4}\right)\right]$ and the average $\mathrm{Co}^{\mathrm{II}}-\mathrm{O}$ bond length is $2.12 \AA$ for $\left[\mathrm{Co}\left(\mathrm{SO}_{4}\right)\left(\mathrm{N}_{2} \mathrm{H}_{4}\right)\right]$, whereas the average $M-\mathrm{N}$ bond lengths involving the $\mathrm{N}$ atom of the hydrazine ligand are

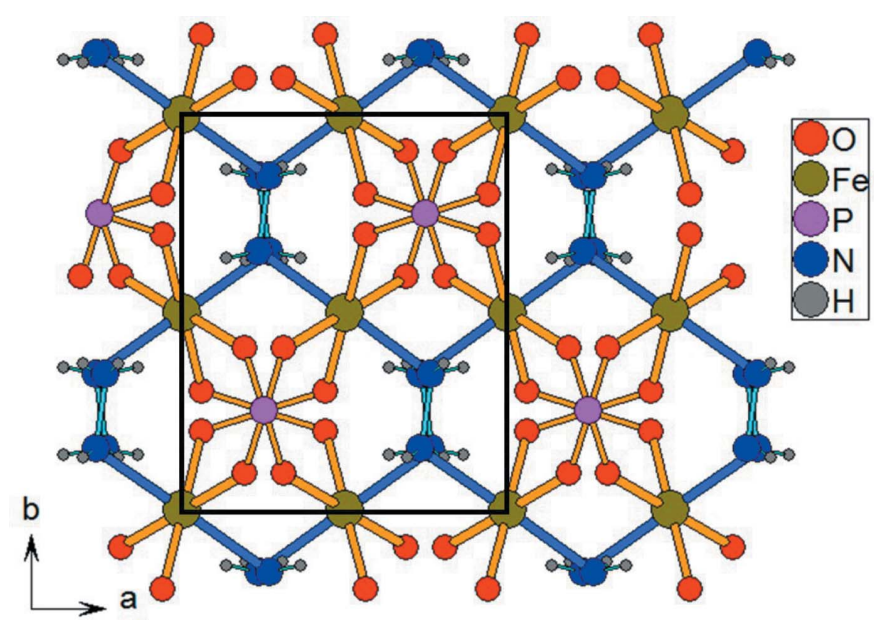

Figure 2

The crystal structure of $\left[\mathrm{Fe}\left(\mathrm{PO}_{4}\right)\left(\mathrm{N}_{2} \mathrm{H}_{4}\right)\right]$ in a projection along [001].

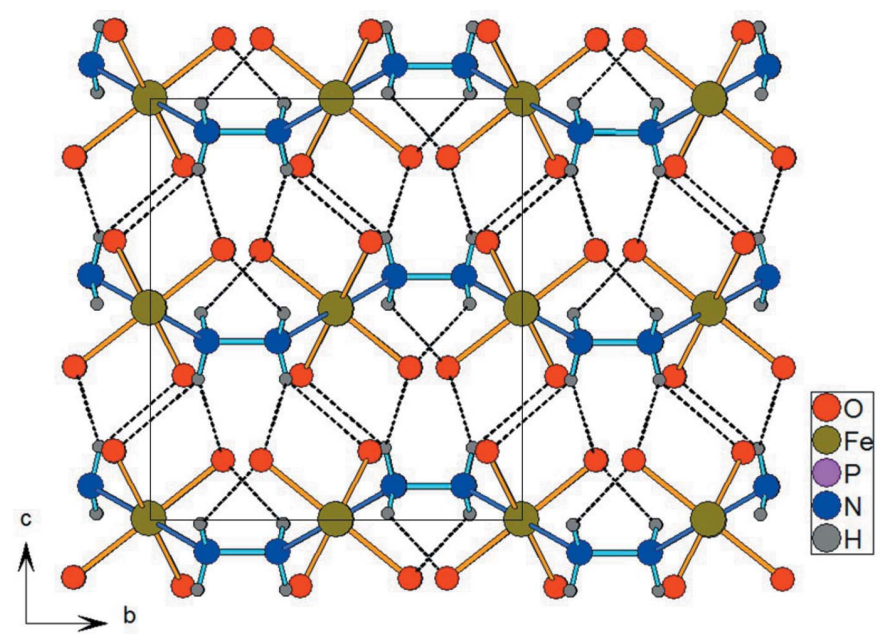

Figure 3

The crystal structure of $\left[\mathrm{Fe}\left(\mathrm{PO}_{4}\right)\left(\mathrm{N}_{2} \mathrm{H}_{4}\right)\right]$ in a projection along [100], emphasizing the hydrogen bonding between the components (black dotted lines). $\mathrm{P}$ atoms have been omitted for clarity.

similar, with values of 2.17 and $2.12 \AA$, respectively. As a consequence, the $\mathrm{FeN}_{2} \mathrm{O}_{4}$ octahedron is more distorted, appearing like an $\mathrm{FeO}_{4}$ square additionally bound by two trans hydrazine ligands in axial positions.

It should be noted that it seems rather surprising to stabilize $\mathrm{Fe}^{\mathrm{III}}$ with hydrazine, since the latter is a powerful reducing agent. Efforts are currently underway to obtain the title compound as a pure phase to perform magnetic measurements. It could be a way, by comparison with the results reported for $\left[\mathrm{Co}\left(\mathrm{SO}_{4}\right)\left(\mathrm{N}_{2} \mathrm{H}_{4}\right)\right]$ (Jia et al., 2011), to study the ability of hydrazine to transmit magnetic coupling.

\section{Supramolecular features}

The three-dimensional framework structure of $\left[\mathrm{Fe}\left(\mathrm{PO}_{4}\right)\right.$ $\left(\mathrm{N}_{2} \mathrm{H}_{4}\right)$ ] is consolidated by $\mathrm{N}-\mathrm{H} \cdots \mathrm{O}$ interactions between the hydrazine ligands and phosphate $\mathrm{O}$ atoms (Fig. 3). One of the two hydrogen bonds is bifurcated. Considering the $\mathrm{N}$... distances and the values of the $\mathrm{N}-\mathrm{H} \cdots$ angles (Table 1 ), this type of hydrogen bonding can be considered as moderately strong.

\section{Synthesis and crystallization}

Iron(II) chloride tetrahydrate (>99.0\%, Sigma-Aldrich), hydrazine monohydrate $(99+\%)$ and $\mathrm{KH}_{2} \mathrm{PO}_{4}$ (both VWR International) were used as received without further purification. Iron(II) chloride tetrahydrate $(2 \mathrm{~g})$ was dissolved in water $(20 \mathrm{ml})$ before adding hydrazine monohydrate $(2 \mathrm{ml})$. The obtained solution was stirred for $5 \mathrm{~min}$. Then, $\mathrm{KH}_{2} \mathrm{PO}_{4}$ $(11.5 \mathrm{~g})$ was added. After $10 \mathrm{~min}$ of stirring for homogenization, the obtained solution $(15 \mathrm{ml})$ was incorporated in a $23 \mathrm{ml}$ autoclave. The autoclave was then heated at $433 \mathrm{~K}$ for $10 \mathrm{~h}$ before being cooled to room temperature at a rate of $10 \mathrm{~K} \mathrm{~h}^{-1}$. The obtained mixture, consiting of orange crystals of the title phase and yellow crystals of an additional phase, was washed with water. The obtained crystals were very small 
(around $20 \mu \mathrm{m}$ ) and well isolated from the others. Details of the composition and structure of the yellow crystals will be described in a forthcoming article.

\section{Refinement details}

Crystal data, data collection and structure refinements are summarized in Table 2. All $\mathrm{H}$ atoms were located in a difference Fourier map and were refined freely with isotropic displacement parameters.

\section{Acknowledgements}

The RS2E (French Network on Electrochemical Energy Storage) and ANR (Labex STORE-EX; grant No. ANR-10LABX-0076) are acknowledged for funding of the X-ray diffractometer.

\section{References}

Barros, N., Airoldi, C., Simoni, J. A., Ramajo, B., Espina, A. \& García, J. R. (2006). Thermochim. Acta, 441, 89-95.

Brandenburg, K. \& Putz, H. (2010). DIAMOND. Crystal Impact GbR, Bonn, Germany.

Bridger, G. L., Salutsky, M. L. \& Starostka, R. W. (1962). J. Agric. Food Chem. 10, 181-188.

Bruker (2013). APEX2, SAINT and SADABS. Bruker-Nonius AXS Inc., Madison, Wisconsin, USA.

Erskine, A. M., Grimm, G. \& Horning, S. C. (1944). Ind. Eng. Chem. 36, 456-460.

Jia, L.-H., Li, R.-Y., Duan, Z.-M., Jiang, S.-D., Wang, B.-W., Wang, Z.-M. \& Gao, S. (2011). Inorg. Chem. 50, 144-154.

Kabbour, H., David, R., Pautrat, A., Koo, H.-J., Whangbo, M.-H., André, G. \& Mentré, O. (2012). Angew. Chem. Int. Ed. 51, 1174511749.

Ouvrard, G., Zerrouki, M., Soudan, P., Lestriez, B., Masquelier, C., Morcrette, M., Hamelet, S., Belin, S., Flank, A. M. \& Baudelet, F. (2013). J. Power Sources, 229, 16-21.
Table 2

Experimental details.

\begin{tabular}{|c|c|}
\hline \multicolumn{2}{|l|}{ Crystal data } \\
\hline Chemical formula & {$\left[\mathrm{Fe}\left(\mathrm{PO}_{4}\right)\left(\mathrm{N}_{2} \mathrm{H}_{4}\right)\right]$} \\
\hline$M_{\mathrm{r}}$ & 182.87 \\
\hline Crystal system, space group & Orthorhombic, Pccn \\
\hline Temperature $(\mathrm{K})$ & 293 \\
\hline$a, b, c(\AA)$ & $\begin{array}{l}6.3114(13), 7.6680(15) \\
\quad 8.6485(18)\end{array}$ \\
\hline$V\left(\AA^{3}\right)$ & $418.55(15)$ \\
\hline$Z$ & 4 \\
\hline Radiation type & Mo $K \alpha$ \\
\hline$\mu\left(\mathrm{mm}^{-1}\right)$ & 3.89 \\
\hline Crystal size $(\mathrm{mm})$ & $0.05 \times 0.03 \times 0.03$ \\
\hline \multicolumn{2}{|l|}{ Data collection } \\
\hline Diffractometer & Bruker APEXII CCD \\
\hline Absorption correction & $\begin{array}{l}\text { Multi-scan (SADABS; Bruker, } \\
\text { 2013) }\end{array}$ \\
\hline$T_{\min }, T_{\max }$ & $0.668,0.746$ \\
\hline $\begin{array}{l}\text { No. of measured, independent and } \\
\text { observed }[I>3 \sigma(I)] \text { reflections }\end{array}$ & $13820,601,457$ \\
\hline$R_{\text {int }}$ & 0.065 \\
\hline$(\sin \theta / \lambda)_{\max }\left(\AA^{-1}\right)$ & 0.717 \\
\hline \multicolumn{2}{|l|}{ Refinement } \\
\hline$R\left[F^{2}>3 \sigma\left(F^{2}\right)\right], w R\left(F^{2}\right), S$ & $0.020,0.027,1.46$ \\
\hline No. of reflections & 601 \\
\hline No. of parameters & 47 \\
\hline $\mathrm{H}$-atom treatment & All $\mathrm{H}$-atom parameters refined \\
\hline$\Delta \rho_{\max }, \Delta \rho_{\min }\left(\mathrm{e} \AA^{-3}\right)$ & $0.40,-0.33$ \\
\hline
\end{tabular}

Computer programs: APEX2and SAINT (Bruker, 2013), SUPERFLIP (Palatinus \& Chapuis, 2007), JANA2006 (Petřičck et al., 2014) and DIAMOND (Brandenburg \& Putz, 2010).

Padhi, A. K., Nanjundaswamy, K. S., Masquelier, C., Okada, S. \& Goodenough, J. B. (1997). J. Electrochem. Soc. 144, 1609-1613.

Palatinus, L. \& Chapuis, G. (2007). J. Appl. Cryst. 40, 786-790.

Petrríček, V., Dušek, M. \& Palatinus, L. (2014). Z. Kristallogr. 229, 345-352.

Ramajo, B., Espina, A., Barros, N. \& García, J. R. (2009). Thermochim. Acta, 487, 60-64. 


\section{supporting information}

Acta Cryst. (2015). E71, 1436-1438 [https://doi.org/10.1107/S2056989015020010]

\section{Crystal structure of hydrazine iron(III) phosphate, the first transition metal} phosphate containing hydrazine

\section{Renald David}

Computing details

Data collection: APEX2 (Bruker, 2013); cell refinement: SAINT (Bruker, 2013); data reduction: SAINT (Bruker, 2013); program(s) used to solve structure: SUPERFLIP (Palatinus \& Chapuis, 2007); program(s) used to refine structure: JANA2006 (Petrićek et al., 2014); molecular graphics: DIAMOND (Brandenburg \& Putz, 2010); software used to prepare material for publication: JANA2006 (Petrićek et al., 2014).

Poly $\left[\left(\mu_{2}\right.\right.$-hydrazine $)\left(\mu_{4}\right.$-phosphato)iron(III)]

\section{Crystal data}

$\left[\mathrm{Fe}\left(\mathrm{PO}_{4}\right)\left(\mathrm{N}_{2} \mathrm{H}_{4}\right)\right]$

$M_{r}=182.87$

Orthorhombic, Pccn

Hall symbol: -P 2ab $2 \mathrm{ac}$

$a=6.3114(13) \AA$

$b=7.6680(15) \AA$

$c=8.6485(18) \AA$

$V=418.55(15) \AA^{3}$

$Z=4$

\section{Data collection}

Bruker APEXII CCD

diffractometer

Radiation source: X-ray tube

phi scan

Absorption correction: multi-scan

(SADABS; Bruker, 2013)

$T_{\text {min }}=0.668, T_{\max }=0.746$

13820 measured reflections

\section{Refinement}

Refinement on $F$

$R[F>3 \sigma(F)]=0.020$

$w R(F)=0.027$

$S=1.46$

601 reflections

47 parameters

0 restraints
$F(000)=364$

$D_{\mathrm{x}}=2.902 \mathrm{Mg} \mathrm{m}^{-3}$

Mo $K \alpha$ radiation, $\lambda=0.71073 \AA$

Cell parameters from 2128 reflections

$\theta=4.2-26.9^{\circ}$

$\mu=3.89 \mathrm{~mm}^{-1}$

$T=293 \mathrm{~K}$

Parallelepiped, orange

$0.05 \times 0.03 \times 0.03 \mathrm{~mm}$

601 independent reflections

457 reflections with $I>3 \sigma(I)$

$R_{\text {int }}=0.065$

$\theta_{\text {max }}=30.6^{\circ}, \theta_{\min }=4.2^{\circ}$

$h=-9 \rightarrow 8$

$k=-10 \rightarrow 10$

$l=-12 \rightarrow 12$

0 constraints

All H-atom parameters refined

Weighting scheme based on measured s.u.'s $w=$ $1 /\left(\sigma^{2}(F)+0.0001 F^{2}\right)$

$(\Delta / \sigma)_{\max }=0.006$

$\Delta \rho_{\max }=0.40 \mathrm{e} \AA^{-3}$

$\Delta \rho_{\min }=-0.33$ e $\AA^{-3}$ 
Fractional atomic coordinates and isotropic or equivalent isotropic displacement parameters $\left(\AA^{2}\right)$

\begin{tabular}{lllll}
\hline & $x$ & $y$ & $z$ & $U_{\text {iso }} * / U_{\text {eq }}$ \\
\hline $\mathrm{O} 1$ & $-0.0604(2)$ & $0.30096(17)$ & $0.36016(16)$ & $0.0093(4)$ \\
$\mathrm{Fe}$ & 0 & 0 & 0 & $0.00652(11)$ \\
$\mathrm{P}$ & -0.25 & 0.25 & $0.25868(8)$ & $0.00567(17)$ \\
$\mathrm{O} 2$ & $-0.1898(2)$ & $0.09269(19)$ & $0.15978(16)$ & $0.0111(4)$ \\
$\mathrm{N}$ & $0.2656(3)$ & $0.1556(2)$ & $0.0781(2)$ & $0.0103(5)$ \\
$\mathrm{H} 1$ & $0.306(4)$ & $0.132(3)$ & $0.169(3)$ & $0.024(7)^{*}$ \\
$\mathrm{H} 2$ & $0.364(4)$ & $0.139(3)$ & $0.012(3)$ & $0.020(7)^{*}$ \\
\hline
\end{tabular}

Atomic displacement parameters $\left(\AA^{2}\right)$

\begin{tabular}{lllllll}
\hline & $U^{11}$ & $U^{22}$ & $U^{33}$ & $U^{12}$ & $U^{13}$ & $U^{23}$ \\
\hline $\mathrm{O} 1$ & $0.0089(6)$ & $0.0090(7)$ & $0.0099(7)$ & $0.0001(5)$ & $-0.0032(5)$ & $-0.0013(5)$ \\
$\mathrm{Fe}$ & $0.00692(19)$ & $0.0059(2)$ & $0.00678(19)$ & $-0.00013(13)$ & $0.00010(16)$ & $-0.00054(16)$ \\
$\mathrm{P}$ & $0.0059(3)$ & $0.0053(3)$ & $0.0057(3)$ & $-0.0001(3)$ & 0 & 0 \\
$\mathrm{O} 2$ & $0.0135(6)$ & $0.0096(7)$ & $0.0101(7)$ & $0.0003(5)$ & $0.0030(6)$ & $-0.0033(6)$ \\
$\mathrm{N}$ & $0.0110(8)$ & $0.0074(8)$ & $0.0123(9)$ & $-0.0017(7)$ & $-0.0012(8)$ & $0.0010(7)$ \\
\hline
\end{tabular}

Geometric parameters $\left(\AA,{ }^{o}\right)$

\begin{tabular}{|c|c|c|c|}
\hline $\mathrm{O} 1-\mathrm{Fe}^{\mathrm{i}}$ & $1.9843(14)$ & $\mathrm{P}-\mathrm{O} 2^{\mathrm{iii}}$ & $1.5268(15)$ \\
\hline $\mathrm{O} 1-\mathrm{P}$ & $1.5346(15)$ & $\mathrm{N}-\mathrm{N}^{\mathrm{iv}}$ & $1.461(2)$ \\
\hline $\mathrm{Fe}-\mathrm{O} 2$ & $1.9621(15)$ & $\mathrm{N}-\mathrm{H} 1$ & $0.85(3)$ \\
\hline $\mathrm{Fe}-\mathrm{O} 2^{\mathrm{ii}}$ & $1.9621(15)$ & $\mathrm{N}-\mathrm{H} 2$ & $0.85(3)$ \\
\hline $\mathrm{P}-\mathrm{O} 2$ & $1.5268(15)$ & & \\
\hline $\mathrm{Fe}^{\mathrm{i}}-\mathrm{O} 1-\mathrm{P}$ & $133.97(8)$ & $\mathrm{O} 1-\mathrm{P}-\mathrm{O} 2^{\mathrm{iii}}$ & $108.25(7)$ \\
\hline $\mathrm{O} 1^{\mathrm{v}}-\mathrm{Fe}-\mathrm{O} 1^{\mathrm{vi}}$ & $180.0(5)$ & $\mathrm{O} 1{ }^{\mathrm{iii}}-\mathrm{P}-\mathrm{O} 2$ & $108.25(7)$ \\
\hline $\mathrm{O} 1^{\mathrm{v}}-\mathrm{Fe}-\mathrm{O} 2$ & $88.09(6)$ & $\mathrm{O} 1^{\mathrm{iii}}-\mathrm{P}-\mathrm{O} 2^{\mathrm{iii}}$ & $109.13(7)$ \\
\hline $\mathrm{O} 1^{\mathrm{v}}-\mathrm{Fe}-\mathrm{O} 2^{\mathrm{ii}}$ & $91.91(6)$ & $\mathrm{O} 2-\mathrm{P}-\mathrm{O} 2^{\mathrm{iii}}$ & $111.85(9)$ \\
\hline $\mathrm{O} 1^{\mathrm{vi}-\mathrm{Fe}-\mathrm{O} 2}$ & $91.91(6)$ & $\mathrm{Fe}-\mathrm{O} 2-\mathrm{P}$ & $146.37(9)$ \\
\hline $\mathrm{O} 1^{\mathrm{vi}}-\mathrm{Fe}-\mathrm{O} 2^{\mathrm{ii}}$ & $88.09(6)$ & $\mathrm{N}^{\mathrm{iv}}-\mathrm{N}-\mathrm{H} 1$ & $104.6(18)$ \\
\hline $\mathrm{O} 2-\mathrm{Fe}-\mathrm{O} 2^{\mathrm{ii}}$ & $180.0(5)$ & $\mathrm{N}^{\mathrm{iv}}-\mathrm{N}-\mathrm{H} 2$ & $104.2(18)$ \\
\hline $\mathrm{O} 1-\mathrm{P}-\mathrm{O} 1^{\mathrm{iii}}$ & $110.23(8)$ & $\mathrm{H} 1-\mathrm{N}-\mathrm{H} 2$ & $112(2)$ \\
\hline $\mathrm{O} 1-\mathrm{P}-\mathrm{O} 2$ & $109.13(7)$ & & \\
\hline \multicolumn{4}{|c|}{ Symmetry codes: (i) $-x, y+1 / 2,-z+1 / 2$; (ii) $-x,-y,-z$; (iii) $-x-1 / 2,-y+1 / 2, z$; (iv) $-x+1 / 2,-y+1 / 2, z$; (v) $-x, y-1 / 2,-z+1 / 2$; (vi) $x,-y+1 / 2, z-1 / 2$} \\
\hline \multicolumn{4}{|c|}{ Hydrogen-bond geometry $\left(\AA,{ }^{\circ}\right)$} \\
\hline$\underline{D-\mathrm{H} \cdots A}$ & $D-\mathrm{H}$ & $\mathrm{H} \cdots A$ & $D-\mathrm{H} \cdots A$ \\
\hline $\mathrm{N}-\mathrm{H} 1 \cdots \mathrm{O} 1^{\text {iv }}$ & $0.85(3)$ & $3.086(2)$ & $144(2)$ \\
\hline $\mathrm{N}-\mathrm{H} 1 \cdots \mathrm{O} 2^{\mathrm{vii}}$ & $0.85(3)$ & $2.974(2)$ & $141(2)$ \\
\hline $\mathrm{N}-\mathrm{H} 2 \cdots \mathrm{O} 1^{\text {viii }}$ & $0.85(3)$ & $2.873(2)$ & $137(2)$ \\
\hline
\end{tabular}

Symmetry codes: (iv) $-x+1 / 2,-y+1 / 2, z$; (vii) $x+1 / 2,-y,-z+1 / 2$; (viii) $-x+1 / 2, y, z-1 / 2$. 